\title{
Espacios Verdes Urbanos Públicos: Ciudadanos y Técnicos Municipales Bases para una Gestión Sostenible
}

\author{
Public Urban Green Spaces: Citizens and Municipal Technicians Bases for \\ Sustainable Management
}

\section{Espaços Verdes Urbanos Públicos: Cidadãos e Técnicos Municipais Bases para a Gestão Sustentável}

\author{
Gonzalo de la Fuente de Val \\ $\mathrm{PhD}$. Ciencias Biológicas \\ Fondo Verde ONG. Huancayo, Perú. \\ Instituto Superior de Medio Ambiente (ISM). Madrid, España \\ gonzalo.delafuente@fondoverde.org \\ (i) https://orcid.org/0000-0002-7540-6238
}

Recibido: agosto 11 de 2021

Aceptado: septiembre 15 de 2021

Publicado: septiembre 20 de 2021

\section{RESUMEN}

El propósito del trabajo fue explorar la opinión, el uso y la frecuentación hacia los Espacios Verdes Urbanos Públicos (EVUPs) por Técnicos Municipales y Ciudadanos. Se diseñó un cuestionario online, obteniendo una muestra de 128 Técnicos Municipales y 1.168 personas de América Latina. Se utilizó el test de TauKendall para establecer relaciones y análisis de regresión logística, para estimar la importancia de las variables asociadas con la frecuentación. Los resultados muestran que los Técnicos demandan formación en paisajismo sostenible. Los Ciudadanos encuestados frecuentan los EVUPs mientras estén bien cuidados y mantenidos. Se concluye que una mayor calidad de los EVUPs ayudaría a mejorar la vida urbana.

Palabras clave: Parques urbanos; frecuentación; paisajismo.

\begin{abstract}
The purpose of the work was to explore the opinion, use and frequency of Public Urban Green Spaces (PUGSs) by municipal technicians and citizens. An online questionnaire was designed, obtaining a sample of 128 municipal technicians and 1,168 people from Latin America. The Tau-Kendall test was used to establish relationships and logistic regression analysis, to estimate the importance of the variables associated with attendance. The results show that technicians demand training in sustainable landscaping. The surveyed citizens frequent PUGSs if they are well cared for and maintained. It is concluded that a higher quality of PUGSs would help to improve urban life.
\end{abstract}

Keywords: Urban parks; frequenting; landscaping. 


\section{RESUMO}

O objetivo do trabalho foi explorar a opinião, uso e frequência dos Espaços Verdes Urbanos Públicos (EVUPS) por Técnicos municipais e cidadãos. Foi elaborado um questionário online, obtendo uma amostra de 128 Técnicos municipais e 1.168 pessoas da América Latina. O teste de Tau-Kendall foi utilizado para estabelecer relações e análise de regressão logística, para estimar a importância das variáveis associadas ao atendimento. Os resultados mostram que os Técnicos exigem capacitação em paisagismo sustentável. Os cidadãos inquiridos frequentam EVUPs desde que sejam bem cuidados e mantidos. Conclui-se que uma maior qualidade dos EVUPs ajudaria a melhorar a vida urbana.

Palavras-chave: Parques urbanos; freqüentando; paisagismo.

\section{INTRODUCCIÓN}

Los Espacios Verdes Urbanos Públicos (EVUPs) son piezas claves de las ciudades (GómezGonçalves, 2013). Los EVUPs, como calles y avenidas arboladas, ríos, plazas ajardinadas, plazas y parques urbanos, otorgan una mejor calidad de vida a las personas en las ciudades (Harnik y Crompton, 2014: Karade et al., 2017). Asimismo, contar con EVUPs seguros e inclusivos es uno de los Objetivos de Desarrollo Sostenible (ODS) promovidos por las Naciones Unidas en el marco de construir ciudades y comunidades sostenibles (ODS11).

La literatura científica muestra que la importancia de los EVUPs radica en que la vegetación mejora la calidad del aire, la estética y amortigua ruidos en la ciudad (Karade et al., 2017; Rey et al., 2018; Kruize et al., 2019), al tiempo que constituyen espacios recreativos que generan bienestar físico y mental con efectos positivos, por ejemplo, frente al estrés, incluso mejorando la calidad del sueño (Palomo, 2003; Marselle et al., 2020; Yang et al., 2020). A su vez, como lugares de integración social, favoreciendo el contacto entre personas de distintas condiciones socioeconómicas (Barrón, 2015).

Los EVUPs, además de servir como refugios y alimento para la avifauna, proporcionan conectividad entre las especies que están dentro y fuera de la ciudad (Faggi y Perepelizin, 2006), cumpliendo un rol significativo en la ecología urbana (Pons, 2016). La conexión en red de EVUPs con corredores verdes favorece la integridad y funcionalidad ecológica de los ecosistemas, al tiempo que contribuyen a la conservación de los hábitats naturales (Burel y Baudry, 2002).
Los EVUPs ayudan en las ciudades a mitigar los efectos del cambio climático. Los bosques, riberas y otros ecosistemas terrestres son sumideros de carbono, dada su capacidad para fijar carbono atmosférico. Un árbol en buen estado puede reducir entre $10 \mathrm{~kg}$ a $30 \mathrm{Kg}$ de $\mathrm{C} 02$, fijando $3 \mathrm{~kg}$ a $6 \mathrm{~kg}$ de carbono (Akbari, 2002; Sanahuja, 2013). Asimismo, una calle con árboles de tamaño regular y otra sin vegetación puede variar de $24^{\circ} \mathrm{C}$ (Falcón, 2007). Lo que ayuda a moderar los extremos de temperatura, reduce el efecto urbano de isla térmica, favoreciendo ahorrar energía y mejorar la calidad del clima en las ciudades (Roy et al., 2012).

Es crucial que los EVUPs sean gestionados adecuadamente para obtener los beneficios esperados de ellos (Karade et al., 2017). Según Mejía y Gómez: "Los espacios verdes han sido utilizados como una forma de contener el crecimiento urbano; por lo tanto, los procesos de expansión recientes pueden comprometer aun más la preservación de áreas verdes, especialmente en ausencia de planificación" (2016, p.4). Actualmente, se anima a que los EVUPs incluyan criterios de sostenibilidad para mejorar la resiliencia de las ciudades frente al cambio global (Flores, 2012b; Pons, 2016; Mengbing y Xiaoling, 2020). La sostenibilidad en los EVUPs implica incluir su tridimensionalidad: lo económico, ambiental y social, en las fases de diseño, construcción y mantenimiento de áreas verdes. Esto es buscando que los EVUPs estén mejor adaptados al medio donde están situados, aplicando conocimientos y utilizando tecnologías para un uso racional del agua, la circularidad, mayor eficiencia energética, uso preferente de especies nativas y otras de bajo mantenimiento, tanto en podas como fertilizantes, entre otros, y un uso social con participación ciudadana en los espacios verdes (Karade et al., 2017; Palomo, 2003). 
La participación ciudadana en los EVUPs da un sentido de pertenencia y favorece que las actuaciones no estén disociadas de la realidad. González apunta que la participación es: "Una forma de intervención social que le permite a los individuos reconocerse como actores que, al compartir una situación determinada, tienen la oportunidad de identificarse a partir de intereses, expectativas y demandas comunes" (1995, p. 23). Como incide De la Fuente-de Val (2010) es un proceso en donde las personas toman parte en las resoluciones de problemas comunes, y dan forma a otro escenario, aportando distintos puntos de vista y compartiendo la responsabilidad en la toma de decisiones. Por lo tanto, permite implicar a los Ciudadanos en los asuntos públicos y a los gobiernos locales un acercamiento mayor hacia la ciudadanía como fórmula para conocer sus necesidades y expectativas (De la Fuente-de Val, 2010). Las decisiones que se toman considerando la participación y el conocimiento de un colectivo son más eficaces y ambientalmente sostenibles que las que no lo son (Sahraoui et al., 2021).

En procesos desarrollo urbano la participación ciudadana resulta fundamental (Sahraoui et al., 2021). Como indica Hernández:

Asegura la viabilidad y factibilidad de planes y propuestas, es decir, se asegura el sustento principal y la razón de las intervenciones en las ciudades; además, los Ciudadanos involucrados adquieren una responsabilidad compartida sobre el éxito de la implementación, así como del seguimiento y continuidad de dichas intervenciones a largo plazo (2007, p.2).

Una correcta planificación involucra a las personas en el diseño, ejecución y gestión de los EVUPs, facilitando así la gobernanza del espacio público. La gobernanza implica la cooperación, negociación y toma de decisiones entre distintos actores públicos, privados y de la sociedad civil (Brenner y De la Vega, 2014). La colaboración y acuerdos entre estos influyen en el buen funcionamiento y calidad de los servicios en los EVUPs. De esta forma, la buena gobernanza promueve y garantiza procesos que facilitan la transparencia, sostenibilidad y equidad de todos (Brenner y De la Vega, 2014). Esto es, toma en cuenta a todos los Ciudadanos sin distinción alguna, entendiendo que no debe haber exclusividad en la toma de decisiones por parte de los gobiernos locales o de aquellos profesionales con competencias, que presuponen por legado la gobernabilidad sobre el territorio urbano (Centelles i Portella, 2006).

Una debilidad de lo anterior es que pocas veces en el proceso de diseño de los EVUPs se considera la opinión de sus usuarios, como tampoco en la mejora y/o rehabilitación de los mismos. La creciente demanda por EVUPs que cubran las necesidades de los usuarios, ha empezado a sensibilizar a los Técnicos Municipales en la necesidad de conocer los gustos y usos que los usuarios tienen de estos espacios (Low et al. 2005). Harnik y Crompton (2014) consideran que son los usuarios de los EVUPs quienes debieran primero expresar sus necesidades, preferencias y requerimientos, ya que hablar de planificación en los EVUPs es hablar de lo que la gente quiere y desea, más no de lo que se debe tener. En concordancia, Harnik (2010) plantea que la planificación de los EVUPs ha ignorado la complejidad de la vida urbana y no puede limitarse al uso de indicadores cuantitativos, por ejemplo, la superficie de los parques, densidad de uso entre otros. A lo cual, Flores profundiza en que los indicadores buscan: "Responder ante organismos internacionales que respecto a dotación de áreas verdes se está cumpliendo con intentar alcanzar el mínimo de superficie recomendado por la OMS, olvidando las necesidades, gustos y preferencias de los citadinos que se verán beneficiados por ella" (2012a, p. 176). Siguiendo a Flores el uso de indicadores ha prevalecido desde finales del siglo XX y responde a un modelo de gestión pública basada en la "simplificación administrativa y una privatización de servicios basada solo en principios económicos de competencia, dinero, incentivos, asignación y eficiencia, dejando de lado los impactos sociales y ambientales que tienen los espacios sobre los citadinos" (ídem).

Entender el uso y percepción de los EVUPs por parte de los Ciudadanos es conocer las variables que explican la motivación y frecuentación a estos espacios. Factores como el género, la distancia, tipo de residencia y niveles de ingreso influyen en la elección de actividades recreativas, frecuencia de visitas, preferencia de días de visita (entre semana o fines de semana) y/o acompañamiento (solo, amigos o familia) (Mäkinen y Tyrväinen, 2008; Karade et al., 2017). De acuerdo con Figueroa (2009) la frecuencia de uso hacia EVUPs es un factor que refuerza el apego social entre los vecinos. 
Forsyth (2003) y Walker (2004) subrayan también la relevancia de conocer los problemas que los usuarios observan de los EVUPs. La seguridad percibida es un factor clave para frecuentar un EVUP, amenazas como vandalismo, retraen el uso y disfrute de estos espacios públicos (Stephens, 2014). De igual forma, EVUPs abandonados, deteriorados, descuidados sin un mantenimiento adecuado desencadenan un aumento en la sensación de inseguridad en los usuarios (Stephens, 2014).

La distancia tiene un rol importante en la visita a los EVUPs. El trabajo de Nilsson y colaboradores (1997) muestra que, cuando la distancia de la vivienda al EVUPs excede los 300 metros, una de cada cuatro personas pospone la visita. Si la distancia es mayor a los 500 metros, el $56 \%$ de personas se abstiene de visitar regularmente ese EVUP (Nilsson et al., 1997). En la mayoría de los casos son más frecuentados los EVUPs que se encuentran al interior de barrios, por ejemplo plazas o pequeñas áreas, a una distancia tal que se pueda acceder en un tiempo no superior a 10 o 15 minutos caminando (Coles y Bussey, 2000; Handley et al., 2003).

Las relaciones y beneficios detectados de los EVUPs corresponden a estudios europeos y/o de América del Norte y pocos de América Latina, siendo una región poco estudiada a pesar del déficit y distribución desigual de los EVUPs en las ciudades (García et al., 2021). En general, los estudios sobre América Latina muestran positivas relaciones entre las características de los EVUPs (por ejemplo, proximidad, tamaño, comodidades) con el uso y los beneficios esperados por los usuarios (Mena et al., 2015; Scopelliti et al., 2016; Salvo et al., 2017). Este estudio se suma a esta literatura emergente. Además, la pandemia del Covid-19 ha puesto de manifiesto la importancia de contar con EVUPs de calidad en las ciudades, como un factor fundamental para ofrecer bienestar, y salud física y mental a los Ciudadanos (HoneyRoses et al., 2020; Ugolini et al., 2020).

En este contexto, el objetivo del trabajo fue conocer qué percepción tienen los Técnicos Municipales y Ciudadanos de los EVUPs, respecto a la función de estos espacios en la ciudad y qué variables pueden influir en la frecuentación de los mismos.

\section{MATERIALES Y MÉTODOS}

El artículo forma parte de un trabajo exploratorio relacionado con el estudio del uso, actitudes y preferencias sobre los EVUPs, el cual fue estructurado en 3 fases (De la Fuente, 2020). En este documento se presentan los resultados preliminares de la primera fase y discutidos a modo general, sin ahondar en el origen de los participantes. Deseamos exponer patrones generales que en siguientes publicaciones se irán presentando y discutiendo con detalle para no sobrecargar los contenidos de las publicaciones. La primera fase se basó en la realización de una encuesta en línea a 128 Técnicos Municipales y 1.168 personas consultadas de América Latina. De las 1.168 respuestas completadas, 40 de ellas $(3,4 \%)$ presentaban datos espurios e incompletos, por lo cual se pasó a eliminarlas, quedando un total de 1.128 cuestionarios válidos.

Junto con colectar datos sociodemográficos, el cuestionario tenía 25 preguntas de selección múltiple y organizada tres en secciones: conociendo el municipio, tus preferencias y el futuro de los EVUPs. Las preguntas de la sección, conociendo el municipio, eran sobre el estado actual y selección de alternativas de intervención y mejora de los EVUPs del municipio en que vive el entrevistado. De igual forma, las preguntas de la sección conociendo tus preferencias eran sobre las actividades de ocio, nivel de frecuentación y grado satisfacción por los EVUPs. Por último, las preguntas de la sección conociendo el futuro eran sobre selección de alternativas de mantenimiento, conservación y gestión de los EVUPs. El cuestionario fue revisado por profesores de la Universidad Autónoma de Baja California, sometido a un grupo de control para corregir errores de interpretación de las preguntas.

A efectos del trabajo, se entiende por EVUPs todos aquellos espacios abiertos, áreas verdes, riberas, quebradas, zonas naturales (o silvestres) y/o recreativas de la ciudad, gestionadas, controladas y mantenidas por parte del municipio; por Ciudadanos, a personas que viven en ciudades y pueblos; y por Técnicos Municipales a personas que trabajan en una entidad municipal relacionada con el diseño, ejecución y mantenimiento de los EVUP. Como parte del estudio fue realizado durante las restricciones de movilidad por la pandemia del Covid-19, el uso y disfrute de los EVUPs por los 
Ciudadanos fue más limitado de lo habitual. Por lo cual, se les pidió a todos los entrevistados que respondieran en función de lo que hacía antes de las restricciones de movilidad para evitar sesgos (Ugolini et al., 2020).

Se construyó una base de datos de entidades municipales, organizaciones civiles, juntas vecinales, etc. de América Latina. El resultado final fue una base de datos con más de 12 mil correos electrónicos. Se realizaron campañas a dichos correos por un tiempo de 3 meses de forma periódica. Complementariamente, se realizaron campañas en redes sociales, como Facebook y Twittter, invitando a participar a Ciudadanos de Latinoamérica.

Antes de procesar los datos se analizó la fiabilidad de las respuestas por secciones utilizando el test Alfa de Cronbach ( $\alpha$ ). Los niveles de fiabilidad fueron $\alpha=0,88$ en conociendo el municipio, $\alpha=0,86$ en conociendo tus preferencias y $\alpha=0,83$ en conociendo el futuro. Los resultados revelan que todas las preguntas son fiables; según Nunnally (1978) un valor Alfa de Cronbach superior a 0,7 se puede considerar confiable.

El cuestionario fue analizado aplicando técnicas monovariantes, describiendo cada una de las preguntas en frecuencias y convertidas en porcentaje. Para observar las relaciones entre la frecuentación de los EVUPs y otras variables se empleó la correlación de Tau-Kendall. Para detectar diferencias se utilizó el test de Kruskal-Wallis para variables con más de dos categorías (la edad, el nivel de estudios, la condición laboral y el tipo de vivienda) y la U de Mann-Whitney para variables de dos categorías (Género y Lugar de Residencia).

Para conocer la importancia de las variables asociadas con la frecuentación se utilizó la regresión logística multinominal (MNL) que permite cuantificar qué variables del estudio afectan y de qué manera a la frecuentación de visita. Se eligió la regresión MNL porque la variable dependiente frecuentación es categórica y permite modelar cómo influye en la probabilidad de aparición de un suceso, en este caso la frecuentación, la presencia o no de diversos factores y el valor de los mismos. Una vez que se establece el modelo de regresión y se calculan los valores estimados de los distintos parámetros, el siguiente paso es verificar que el modelo reúne los requisitos estadísticos. Su uso ha resultado adecuado en estudios similares sobre EVUPs (Burrows et al., 2018; Flores, 2012b) más información sobre la regresión MNL ver Pando y San Martín (2004). Para el cálculo de estas pruebas estadísticas se utilizó el paquete estadístico IBM SPSS Statistics for Windows (IBM, 2020). El nivel de significación fijado fue $\mathrm{p}<0,05$ y $\mathrm{p}<0,01$.

\section{RESULTADOS Y DISCUSIÓN}

Situación y futuro de los EVUPS según los Técnicos Municipales. 128 Técnicos han completado el cuestionario, procedentes de 128 municipios de 17 países de América Latina (ver Tabla 1). En su mayoría profesionales adultos, con edad superior a los 35 años. El $10 \%$ son paisajistas y la gran mayoría arquitectos e ingenieros forestales, agrónomos, biólogos entre otros. La mitad de los Técnicos tienen estudios de posgrado; de ellos solo el $23 \%$ tiene especialización en paisajismo o en jardinería.

Tabla 1. País de origen de los Técnicos Municipales ( $\mathrm{N}=128)$.

\begin{tabular}{cc}
\hline País & $\%$ \\
\hline Chile & 17,2 \\
Colombia & 13,3 \\
México & 10,9 \\
Argentina & 9,4 \\
Bolivia & 5,5 \\
Perú & 5,5 \\
Nicaragua & 4,7 \\
Uruguay & 4,7 \\
Paraguay & 3,9 \\
República Dominicana & 3,9 \\
Brasil & 3,9 \\
Costa Rica & 3,1 \\
Cuba & 3,1 \\
El Salvador & 3,1 \\
Honduras & 3,1 \\
Ecuador & 2,3 \\
Guatemala & 2,3 \\
\hline
\end{tabular}

Fuente: Elaboración propia

Al ser preguntados sobre los EVUPs que gestionan en su municipio, más de la mitad indican: parques urbanos, plazas, monumentos, parques vecinales, etc. (ver Tabla 2). Consultados si el municipio cuenta con alguna normativa sobre los EVUPs, el $65,6 \%$ indica que no poseen ordenanza que ordene, gestione y proteja los parques y jardines públicos. Además, comprobamos que cuando existe alguna normativa, no hay capacitación e información 
al respecto. Esta situación podría condicionar a los profesionales en su rol de fiscalización de los asuntos públicos por desconocimiento de la normativa de aplicación si no también relegarla a situaciones meramente reactivas (Cornejo, 2016). En lugar de tener un carácter preventivo de mayor valor que potencie la protección de los EVUPs en la ciudad.

Tabla 2. Opinión sobre los EVUPs según Técnicos Municipales $(\mathrm{N}=128)$.

\begin{tabular}{|c|c|}
\hline Categoría & $\%$ \\
\hline \multicolumn{2}{|l|}{ Tipología de EVUPs } \\
\hline Parques municipal & 69,5 \\
\hline Plazas & 66,3 \\
\hline Avenidas y calles arboladas & 63,0 \\
\hline Parque vecinal & 59,8 \\
\hline Glorietas, rotondas & 40,0 \\
\hline Zonas forestales & 29,5 \\
\hline \multicolumn{2}{|l|}{ Función de los EVUPs } \\
\hline Calidad de vida & 97,7 \\
\hline Calidad estética & 92,0 \\
\hline Control de la contaminación & 90,3 \\
\hline Contacto naturaleza & 90,0 \\
\hline Salud publica & 90,6 \\
\hline \multicolumn{2}{|l|}{ Como mejorar los EVUPs } \\
\hline $\begin{array}{l}\text { Criterios ambientales } \\
\end{array}$ & 90,0 \\
\hline Reducción de huella carbono & 90,5 \\
\hline $\begin{array}{l}\text { Planes de re vegetación } \\
\text { Proteger parques frente a expansión }\end{array}$ & 90,0 \\
\hline & 90,2 \\
\hline Seguridad ciudadana & 85,0 \\
\hline Reducir consumo de agua y luz & 85,0 \\
\hline Huertos comunitarios & 84,9 \\
\hline $\begin{array}{l}\text { municipales } \\
\text { mulocios }\end{array}$ & 83,0 \\
\hline
\end{tabular}

Fuente: Elaboración propia

Preguntados las funciones que los EVUPs cumplen en la ciudad, según cuatro alternativas propuestas, los Técnicos apuntan mayoritariamente a mejorar la calidad estética de la ciudad, controlar la contaminación atmosférica, facilitar el contacto con la naturaleza y brindar salud pública (ver Tabla 2). Asimismo, indican que una mayor cantidad de EVUPs ayudaría a mejorar la calidad de vida de los Ciudadanos (97,7\%). En relación en cómo se pueden mejorar los EVUPs, según ocho alternativas propuestas, concuerdan en introducir criterios ambientales en el diseño y mantenimiento de los parques y jardines, reducir la huella de carbono e incentivar plantaciones con vegetación natural (Tabla 2).
Una gestión idónea de los EVUPs, requiere de profesionales preparados que permitan realizar acciones eficaces (Falcón, 2007). Comprobamos que la formación es baja. Menos de la mitad tiene formación formal en paisajismo. Es decir, estudios académicos relacionados con la planificación, diseño y construcción de áreas verdes. De igual manera, menos de la mitad tiene formación formal en cuestiones técnicas de jardinería pública. Es decir, carecen de conocimiento en mantener y conservar las áreas verdes y sus elementos en buenas condiciones.

La amplia mayoría de los Técnicos (97,3\%) expresa su acuerdo en que se debe mejorar su formación para que puedan mantener en óptimas condiciones los EVUPs. Esto arroja una pregunta, ¿A dónde dirigir los esfuerzos para mejorar las competencias y habilidades de los Técnicos municipales? Según los encuestados, en relación con los estudios de paisajismo, más de la mitad indican que se debe fomentar principalmente estudios en planificación de áreas verdes junto con diseño de infraestructuras verdes. Así como en sistemas urbanos de drenaje sostenible y también en planes de recuperación de espacios verdes (Tabla 3). En relación con los estudios más Técnicos de jardinería, apuntan a la formación en Control de plagas, Manejo de podas, Jardinería ecológica y Producción de plantas ornamentales y forestales (Tabla 3).

Tabla 3. Tipo de formación demandada según los Técnicos Municipales ( $N=128)$.

\begin{tabular}{lc}
\hline \multicolumn{1}{c}{ Categoría } & $\%$ \\
\hline Formación en paisajismo & \\
\hline Planificación de EVUPs & 72,0 \\
Diseño de infraestructuras verdes & 72,7 \\
Sistemas urbanos de drenaje sostenible & 62,7 \\
Planes de recuperación de EVUPS & 61,6 \\
Gestión del arbolado urbano & 58,0 \\
\hline Formación en jardinería & 54,0 \\
\hline \multicolumn{2}{c}{ Control de plagas } \\
Manejo de podas & 53,6 \\
Jardinería ecológica & 53,3 \\
Producción de plantas ornamentales y forestales & 53,0 \\
Técnicas de reutilización de residuos vegetales urbanos & 49,2 \\
\hline
\end{tabular}

Fuente: Elaboración propia

En resumen, se constata que los Técnicos demandan una formación más actualizada acorde a los nuevos tiempos que requiere la creación y mantenimiento de EVUPs más sostenibles, saludables y medioambientalmente responsables (Karade et al., 2017; Palomo, 2003). En los cuales, 
haya una mayor eficiencia energética, reducción drástica del uso de fitosanitarios y fertilizantes, mejor gestión de los restos de materias orgánicas, del recurso hídrico entre otros (Palomo, 2003).

Preguntados cómo visualizan el futuro, los Técnicos concuerdan que la biodiversidad jugará un papel significativo en un escenario 2030 en el marco del ODS "ciudades y comunidades sostenibles" de la ONU. Más de la mitad opinan que será una tendencia en aumento el uso de especies herbáceas nativas de bajo consumo de agua buscando un menor mantenimiento de los EVUPs. Así como incluir funciones ecológicas en el diseño de los parques con diseño más Naturalizados de las plantaciones vegetales (ver Figura 1). A tenor de las respuestas, se aprecia que para los Técnicos la vegetación ha dejado de ser algo meramente ornamental para convertirse en un elemento con funciones ecológicas (Falcón, 2007; Karade et al., 2017).

\section{Uso de especies herbáceas nativas de bajo consumo de agua en grandes extensiones para menor mantenimiento}

\section{Funciones ecológicas en el diseño de los parques: fomento de la biodiversidad, diseīo sensible al agua, y similares.}
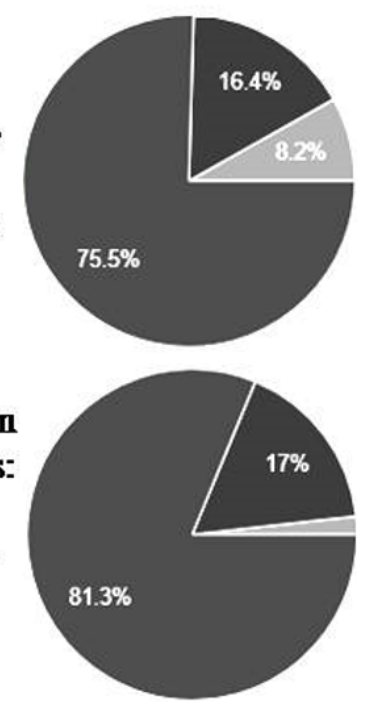

\section{Uso de Sistemas Urbanos de Drenaje Sostenible en parques existentes, nuevos o rehabilitaciones}

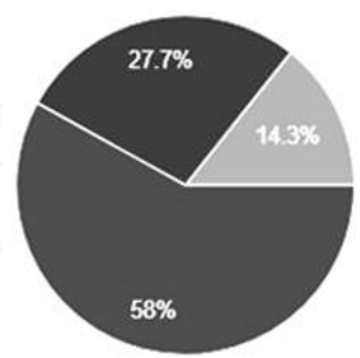

Si, será una tendencia en aumento

Seguirá como en la actualidad No, será algo excepcional

Figura 1. Respuestas sobre la gestión futura de los EVUPs según Técnicos Municipales ( $N=128)$

Fuente: elaboración propia.
A su vez, más de la mitad estima que será una tendencia en aumento promover el confort ambiental, mediante el aumento de sombras y elementos de agua. La mayoría de las actividades de esparcimiento y ocio en los parques se desarrollan al aire libre; por lo cual, proveer equipamientos que brinden frescor y resguardo del sol es esencial para satisfacer las necesidades de los usuarios (Laurie, 1983) y como acción de adaptación de los espacios públicos urbanos a la crisis del cambio climático (Krellenberg et al., 2013).

Para lograr una eficiente gestión de los EVUPs es imprescindible que los Técnicos puedan colaborar con los Ciudadanos haciéndoles corresponsables de su conservación y protección (Kruize et al., 2019). Por ello, es positivo que más de la mitad consideran que se debe apoyar a las asociaciones vecinales para que tengan un papel más activo en la gestión de los EVUPs (Figura 2).

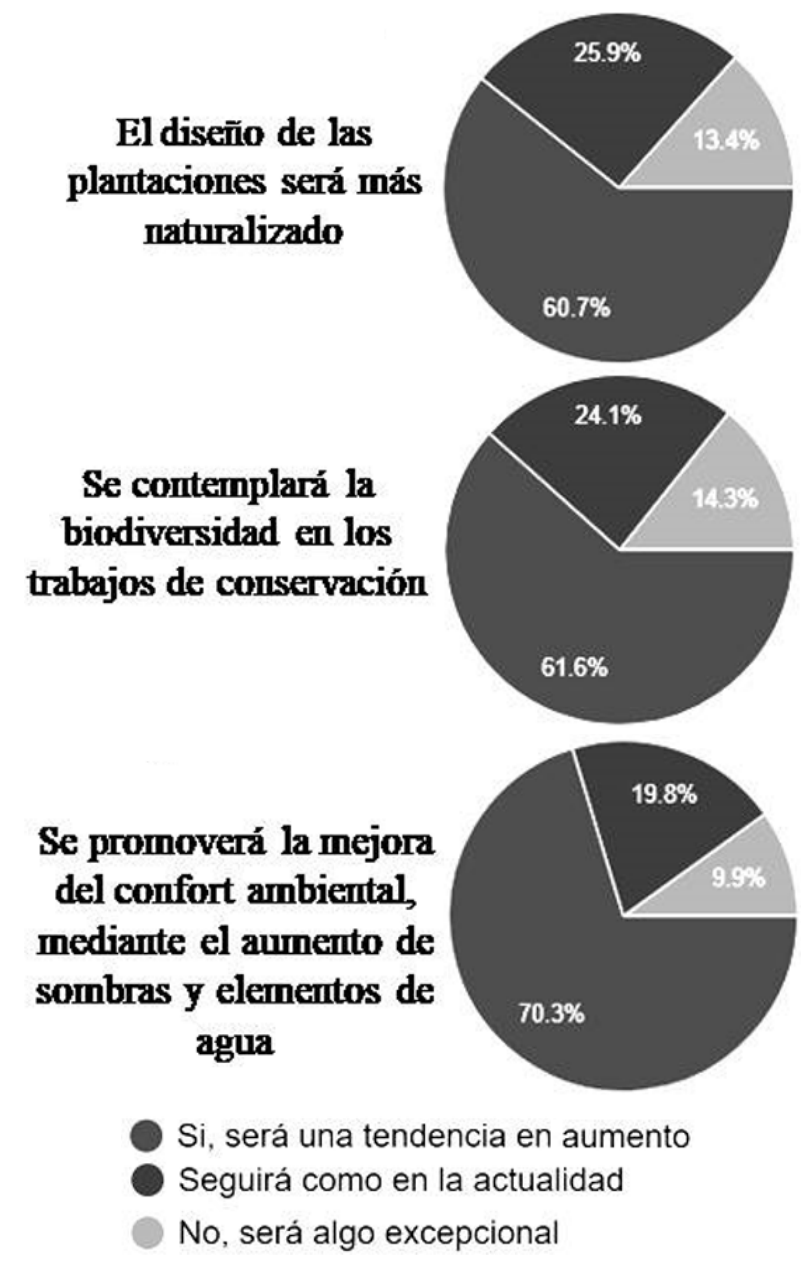

Figura 2. Respuestas sobre la gestión futura de los EVUPS, según Técnicos Municipales $(N=128)$.

Fuente: elaboración propia. 
En esa línea, consultados de cara al futuro qué características paisajísticas demandarán los Ciudadanos de los EVUPs, los Técnicos apuntan a que ofrezcan: tranquilidad, mayor contacto con la naturaleza, amplias superficies para reuniones sociales y los niños puedan jugar libremente.

Es interesante ver que los Técnicos destaquen la importancia de no solo ofrecer mayor naturaleza y espacios para interactuar socialmente; si no también que inspiren calidad emocional. Recuperando la importancia de la tranquilidad como componente esencial del espacio público que busca proveer bienestar y salud mental. Brindando sensación de paz, aislamiento y silencio a las personas agotadas de la estresante vida urbana (Vargas y Roldán, 2018).

Situación y futuro de los EVUPS según los Ciudadanos. 1.128 personas completaron el cuestionario, provienen de 17 países representando a 660 municipios de ciudades de América Latina. La mayoría son jóvenes-adultos, con estudios secundarios y universitarios, en activo, viven principalmente en ciudades, en casas con jardín. La proporción entre mujeres y hombres es similar (Tabla 4).

Al revisar las respuestas que EVUPs existen en el municipio, la mayoritaria cuenta con parques urbanos, plazas, avenidas y calles arboladas (Tabla 5). Sobre los beneficios que aportan los EVUPs a su municipio, destacan ser un pulmón verde, ofrecer contacto con la naturaleza y como espacio para el esparcimiento, recreación y de relajación para combatir el estrés urbano. Todos coinciden que una mayor cantidad de parques y jardines en la ciudad ayudaría a mejorar su calidad de vida (98,2\%) (Tabla 5). Se observa que los EVUPs, a pesar de estar inmersos en un contexto urbano, son percibidos por los Ciudadanos como lugares que brindan beneficios de carácter medioambiental, social y emocional.

Hacia el futuro, todos apoyan las seis alternativas propuestas para mejorar los EVUPs del lugar en que viven. El 95\%, recogiendo las opciones de acuerdo y totalmente de acuerdo, son favorables a introducir criterios ambientales en el diseño y mantenimiento de parques y jardines, dotar de mayor seguridad y crear más parques urbanos. Asimismo, están de acuerdo en incluir a los vecinos en el diseño de plazas y parques e incentivar los huertos urbanos de uso comunitario como acciones destacadas de adaptación al cambio climático y la resiliencia urbana (Tabla 6).
Tabla 4. Descripción de variables socio demográficas de los Ciudadanos $(\mathrm{N}=1128)$.

\begin{tabular}{|c|c|c|}
\hline Variable & Categoría & $\%$ \\
\hline \multirow{16}{*}{ Países } & Colombia & 17,6 \\
\hline & Brasil & 13,2 \\
\hline & Chile & 11,3 \\
\hline & México & 10,8 \\
\hline & Argentina & 7,5 \\
\hline & Costa Rica & 5,9 \\
\hline & Ecuador & 5,9 \\
\hline & Guatemala & 5,3 \\
\hline & Bolivia & 3,9 \\
\hline & Perú & 3,7 \\
\hline & Uruguay & 2,6 \\
\hline & Panamá & 2,6 \\
\hline & República Dominicana & 2,0 \\
\hline & Paraguay & 1,7 \\
\hline & Honduras & 1,6 \\
\hline & Nicaragua & 1,5 \\
\hline \multirow{2}{*}{ Género } & Femenino & 56,9 \\
\hline & Hombre & 43,0 \\
\hline \multirow{5}{*}{ Edad } & menos 18 años & 0,8 \\
\hline & 18 -30 años & 22,4 \\
\hline & $31-45$ años & 32,1 \\
\hline & 45- 65 años & 39,3 \\
\hline & más de 66 años & 4,7 \\
\hline \multirow{4}{*}{ Formación } & Sin estudios & 10,8 \\
\hline & Secundaria & 44,7 \\
\hline & Técnica & 15,5 \\
\hline & Universitaria & 29,0 \\
\hline \multirow{3}{*}{ Estado Laboral } & Estudiante & 9,5 \\
\hline & Trabaja & 82,2 \\
\hline & Desempleado & 4,7 \\
\hline \multirow{4}{*}{ Vivienda } & Casa con jardín & 53,3 \\
\hline & Casa sin jardín & 14,0 \\
\hline & Departamento con jardín & 14,2 \\
\hline & Departamento sin jardín & 17,5 \\
\hline \multirow{2}{*}{ Lugar de residencia } & Ciudad & 85,4 \\
\hline & Fuera de la ciudad (zona rural) & 14,6 \\
\hline
\end{tabular}

Fuente: Elaboración propia

Aparece, en una situación intermedia, la medida de reducción de consumo de agua y luz en los parques y jardines. Aquí cabe destacar que casi el $30 \%$ de las opiniones se muestran desfavorables a un uso más eficiente del consumo de agua y luz. Al analizar estas respuestas, podría estar relacionado con algún uso del agua potable de los EVUPs. De hecho, algunos estudios han encontrado que el agua potable de los parques y plazas es un bien que se utiliza en circunstancias especiales como corte de suministros, etc. (Reyes-Päcke et al., 2014). Por lo cual, este resultado puede sugerir que cualquier medida de restricción o de acceso al uso del potable es contemplado como una situación negativa más allá de lo positivo que pueda ser en la búsqueda de una gestión eficiente del consumo de agua. 
Tabla 5. Opinión sobre los EVUPs del municipio en que viven los Ciudadanos $(\mathrm{N}=1128)$.

\begin{tabular}{|c|c|}
\hline Categoría & $\%$ \\
\hline \multicolumn{2}{|l|}{ Tipología de EVUPs } \\
\hline Parques urbanos & 74,8 \\
\hline Plazas & 54,5 \\
\hline Calles y avenidas arboladas & 51,4 \\
\hline Campos deportivos públicos & 38,7 \\
\hline Áreas forestales & 29,5 \\
\hline Paseos peatonales arbolados & 15,8 \\
\hline Glorietas, rotondas & 15,2 \\
\hline Huertos comunitarios & 11,0 \\
\hline \multicolumn{2}{|l|}{ Beneficios de los EVUPs } \\
\hline Pulmón verde frente a la contaminación atmosférica & 64,8 \\
\hline Contacto con la naturaleza & 56,8 \\
\hline Esparcimiento y recreación & 56,0 \\
\hline Relajación/estrés & 45,7 \\
\hline Actividad física al aire libre & 34,9 \\
\hline Acceso libre y gratuito & 31,8 \\
\hline Salud & 26,3 \\
\hline Espacio llevar a los niños & 21,1 \\
\hline Contacto social & 14,8 \\
\hline \multicolumn{2}{|l|}{ Mayor cantidad de EVUPs en la ciudad } \\
\hline $\mathrm{Si}$ & 98,2 \\
\hline No & 1,8 \\
\hline
\end{tabular}

El análisis de varianza muestra diferencias significativas en cinco de las seis alternativas, no resultando significativo la formación académica (Tabla 7). Se comprueba que no hay discrepancias en crear más parques urbanos, siendo una acción unánime no sujeto a variaciones por factores personales de los encuestados.

La creación de huertos comunitarios es la opción que más diferencias presenta. Las mujeres se muestran más favorables a ellos que los hombres. Estudios han mostrado que una mayor presencia y asiduidad de mujeres en huertos urbanos está relacionada, en parte, por la posibilidad de cultivar alimentos propios para así complementar la dieta familiar. Los resultados positivos de producción de alimentos para autoconsumo en zonas urbanas, vendría avalar la valoración de las mujeres encuestadas por iniciativas de agricultura urbana. De hecho, en la mayoría de proyectos de la FAO en Latinoamérica de huertos comunitario urbanos y periurbanos participan mujeres (FAO, 2014).

Tabla 6. Porcentaje de aceptación a las 6 alternativas de mejora de los EVUPs según la opinión de los Ciudadanos $(\mathrm{N}=1128)$.

\begin{tabular}{|c|c|c|c|c|c|c|}
\hline & $\begin{array}{c}\text { Totalmente } \\
\text { en desacuerdo }\end{array}$ & $\begin{array}{c}\text { En } \\
\text { Desacuerdo }\end{array}$ & Indiferente & De Acuerdo & $\begin{array}{l}\text { Totalmente } \\
\text { de acuerdo }\end{array}$ & $\%$ \\
\hline Crear parques y jardines & 1,0 & 1,4 & 1,4 & 16,6 & 79,6 & 100 \\
\hline Reducir consumo de agua y luz en parques & 7,5 & 21,6 & 16,1 & 31,2 & 23,7 & 100 \\
\hline Incluir a los vecinos en el diseño de parques & 1,1 & 4,2 & 10,3 & 32,8 & 51,6 & 100 \\
\hline Mayor seguridad ciudadana en parques & 1,2 & 1,4 & 4,3 & 27,6 & 65,5 & 100 \\
\hline Crear huertos comunitarios & 2,1 & 3,1 & 11,0 & 28,6 & 55,2 & 100 \\
\hline Introducir criterios ambientales en diseño de parques & 1,0 & 0,4 & 1,6 & 11,8 & 85,3 & 100 \\
\hline
\end{tabular}

Fuente: Elaboración propia

Tabla 7. Diferencias en los beneficios de los EVUPs según características socio demográfica de los Ciudadanos (N=1128).

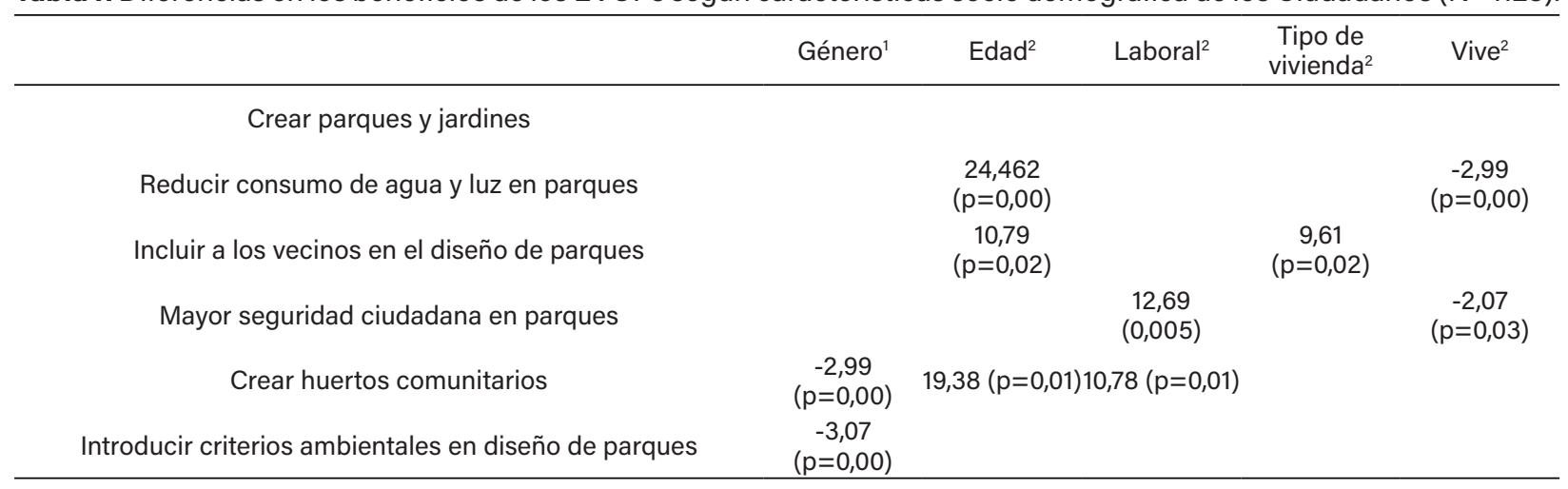

1: Análisis de Kruskal-Wallis; 2: Análisis de U de Mann-Whitney

Valor en cursiva: $\mathrm{p}$ (significación estadística) $<0,05$.

Fuente: Elaboración propia 
Las mujeres están más dispuestas a introducir criterios ambientales en el diseño de los espacios verdes que los hombres. Las mujeres y hombres interactúan de manera diferente con el medio ambiente, diversos estudios apuntan que las mujeres contaminan menos que los hombres, y muestran comportamientos menos dañinos con el entorno (Emakunde, 2014: Kawgan-Kagan, 2020). Las mujeres son más partidarias de establecer restricciones y obligaciones a favor de un consumo y una gestión sostenible de los recursos naturales (Emakunde, 2014; Kawgan-Kagan, 2020). Esto explicaría el apoyo favorable de las mujeres por la adopción de criterios ambientales en el diseño con respecto a los hombres.

Se aprecia que la creación de huertos comunitarios es también una acción muy valorada por estudiantes y desempleados. Mientras tanto, las personas mayores se muestran menos favorables a crear huertos comunitarios que las personas más jóvenes y adultas. Los huertos comunitarios urbanos también involucran un trabajo físico de mantenimiento diario de las parcelas por los hortelanos. Las personas mayores tienden a preferir actividades más pasivas que no requieran un excesivo uso de la fuerza física que las personas más jóvenes más predispuestas de forma natural al desarrollo físico de actividades al aire libre. Esto explicaría el apoyo más favorable de las personas más jóvenes por los huertos. No obstante, los huertos comunitarios son también un espacio para los adultos mayores, solo se debe de proveer de diseños adaptados a sus características físicas, necesidades específicas e inclusivas para personas con movilidad reducida.

A los estudiantes les resulta importante dotar de mayor seguridad a los EVUPs que a los demás grupos de edad. Al igual que las personas que viven en la ciudad, la seguridad es un asunto relevante. La sensación de seguridad y de vigilancia son factores claves para visitar los EVUPs; aquellos que sean percibidos como inseguros pierden atractivo (Stephens, 2014). La problemática de la seguridad ciudadana se agudiza en entornos urbanos más expuestos a situaciones de inseguridad, como vandalismo, robo o violencia personal, fomentando el miedo, sea real o imaginario, al espacio público. A causa de esto, la utilización del espacio disminuye y por extensión la calidad (Stephens, 2014). De ahí, la importancia manifestada por los Ciudadanos, en este trabajo, de dotar de mayor seguridad a los espacios públicos permitiendo así un mayor control sobre el mismo. Como bien expresa William Whyte: "la mejor forma de eliminar todo aquello que sea indeseable es crear lugares muy atractivos para tantas personas como sea posible" (1980, p. 63).

Las personas que viven fuera de la ciudad son más favorables a la reducción del consumo de agua y luz que aquellos que viven en las ciudades. Asimismo, se constata que la reducción del consumo de agua es una opción de mayor importancia para los adultos jóvenes que para las personas mayores y jóvenes. Las ciudades y áreas urbanas se ven expuestas a muchos problemas ambientales, entre ellos, la falta de recursos hídricos (Pons, 2016). Muchas veces el uso poco eficiente del agua (consumo excesivo y despilfarro) tiene que ver, en parte, con una deficiente gestión doméstica (Gogiel, 2011). Según, Gogiel (Ídem) el fomento de la concienciación social en el ámbito doméstico hacia un cambio de actitud es un factor clave para una mejor gestión de este recurso. Así, la educación ambiental ayuda a que los Ciudadanos tomen conciencia de que cada acción que realizan en su vida cotidiana tiene un impacto en el entorno. De acuerdo con Corral-Verdugo (2004) la carencia de un recurso natural incrementa la motivación para cuidarlo. De hecho, el estudio de Gogiel (2011) muestra que la ciudad de Barcelona, a partir de las sequías que han ocurrido desde 2005, por la presión de los medios de comunicación las personas han empezado a consumir menos y partir de esto se han acostumbrado y han mantenido bajos niveles de consumo.

Los resultados obtenidos sugieren la importancia de que los Ciudadanos tomen conciencia y adopten una actitud de respeto hacia la naturaleza. Entendiendo que la calidad de vida urbana depende de la integridad ecológica de los ecosistemas que sustentan las ciudades (Pons, 2016). Cualquier programa de conservación de la naturaleza debe asegurar un sentido de pertenencia y participación activa de la comunidad (Kinne, 1997). Por lo tanto, es indispensable producir programas de educación ambientales participativos en donde los Ciudadanos aprendan a tener una convivencia respetuosa y responsable con su ambiente.

Las personas que viven en departamentos sin jardín son más favorables hacer partícipe a los vecinos en el diseño de EVUPs. La falta de participación ciudadana en la gestión de los EVUPs representa obstáculos hacia un desarrollo en el cual las posibilidades de encuentro y sociabilidad vayan 
en aumento. De ahí, la importancia manifestada, en este trabajo, por los Ciudadanos de incluir a los vecinos en el diseño de los parques para crear espacio que respondan a sus necesidades colectivas favoreciendo así un sentimiento de pertenencia al sentirse identificado con el espacio público. Por cuanto, proyectos paisajísticos que han propuesto equipamientos y funciones de una manera arbitraria no contando con la opinión de los vecinos han carecido de aceptación social (Márquez, 2009). En un diseño participativo, los Ciudadanos son parte del equipo de diseño, aportando sus necesidades, expectativas, etc. La colaboración entre Ciudadanos y Técnicos fortalece el diseño final. Ya que tras su construcción no solo será un espacio agradable para frecuentar sino que también un lugar en donde los Ciudadanos se sienten identificados (Márquez, 2009).

Comparando la opinión entre los Técnicos y Ciudadanos. El análisis de varianza muestra que los Ciudadanos y Técnicos concuerdan en qué se debe aumentar la seguridad y adoptar criterios ambientales en el diseño y mantenimiento de los EVUPs. En cambio, a los Técnicos otorgan mayor importancia a reducir el consumo de agua y luz en los EVUPs; mientras los Ciudadanos a la creación de nuevos espacios verdes que a los Técnicos municipales.

Como en otros estudios frente a los EVUPs existen expectativas distintas (Karade et al., 2017). Los Ciudadanos valoran más la naturaleza producto de la necesidad de mejorar la calidad de vida y bienestar de sus barrios por el aporte positivo que significa la presencia de vegetación en sus parques y calles (Stessens et al., 2020). Mientras tanto, siendo la naturaleza un valor relevante para los Técnicos, muchas veces sus opiniones están matizadas por motivaciones profesionales (de la Fuente-de Val, 2002). Es decir, están influidas por conocimientos académicos y propios de la actividad profesional que hacen modular sus opiniones (de la Fuente-de Val, 2002). Esto se aprecia en la importancia que tiene para los Técnicos acometer un uso eficiente del agua y ahorro en el gasto energético en un escenario de cambio climático (Krellenberg et al., 2013).

Uso y Frecuentación de los EVUPs. Los EVUPs que más visitan son, principalmente, parques urbanos, plazas, avenidas y calles arboladas. Preguntados a qué distancia está el EVUP que visitan regularmente, la mayoría indican entre 500 y 1000 m de la vivienda (ver Tabla 8), acuden más de dos veces por semana en distintas franjas horarias. Una parte de ellos va por la tarde y otros por la mañana, y el resto a cualquier hora del día. El tiempo de estancia es alto, la mayoría está de 1 a más de 2 horas; una parte muy pequeña, menos del $20 \%$, suele estar poco tiempo alrededor de media hora. Principalmente, van caminando, y unos pocos en vehículos propios o en bicicleta. La mayoría van solos o acompañados con amigos o familiares de preferencia realizan actividades pasivas de bajo impacto como son caminar, descansar, etc.

Así, la cercanía, la frecuencia, el tiempo de permanencia y el uso preferente de actividades de ocio pasivas son factores que nos muestra la importancia de los EVUPs para los usuarios entrevistados, haciendo un uso a escala de barrio de los EVUPs (Madrid, 2010, Gómez y Mesa, 2015, Gómez-Gonçalves et al., 2018).

Tabla 8. Compartimientos de visita a los EVUPs más frecuentados por los Ciudadanos $(N=1128)$.

\begin{tabular}{|c|c|c|}
\hline & Categoría & $\%$ \\
\hline \multirow[t]{3}{*}{ Tipo de EVUPs } & Parques urbanos & 65,0 \\
\hline & Plazas & 45,0 \\
\hline & Calles y avenidas arboladas & 35,0 \\
\hline \multirow[t]{4}{*}{ Distancia } & $<500 m$ & 40,09 \\
\hline & $500-1000 \mathrm{~m}$ & 25,30 \\
\hline & $1000-3000 \mathrm{~m}$ & 18,10 \\
\hline & Más de 3000 m & 15,70 \\
\hline \multirow[t]{4}{*}{ Frecuencia } & Semanal & 46,5 \\
\hline & Varias veces al mes & 20,3 \\
\hline & Alguna veces al mes & 25,4 \\
\hline & Una vez al mes o casi nunca & 7,6 \\
\hline \multirow[t]{4}{*}{ Momento del día } & Mañana & 32,0 \\
\hline & Tarde & 37,5 \\
\hline & Noche & 4,8 \\
\hline & A cualquier hora & 25,7 \\
\hline \multirow[t]{4}{*}{ Tiempo } & $<30$ & 20,0 \\
\hline & $1 \mathrm{~h}$ & 36,8 \\
\hline & 1- $2 \mathrm{hrs}$ & 34,5 \\
\hline & +2 horas & 8,7 \\
\hline \multirow[t]{3}{*}{ Con quien } & Solo & 42,0 \\
\hline & Amigos & 37,0 \\
\hline & Familia & 34,0 \\
\hline \multirow{4}{*}{$\begin{array}{l}\text { Medio de } \\
\text { transporte }\end{array}$} & Caminando & 71,0 \\
\hline & Bicicleta & 17,0 \\
\hline & Vehículo propio & 30,0 \\
\hline & Transporte publico & 12,0 \\
\hline \multirow[t]{5}{*}{ Actividades } & Caminar, pasear & 88,0 \\
\hline & Deporte & 16,7 \\
\hline & Jugar con hijos & 25,6 \\
\hline & Reunirse amigos & 39,0 \\
\hline & Relajarse, descansar & 39,0 \\
\hline
\end{tabular}

Fuente: Elaboración propia 
Siguiendo a Gómez-Gonçalves et al. (2018) si los usuarios frecuentan los EVUPs que se encuentran cerca de su vivienda, habrá que cuidar su mantenimiento con estándares mínimos de calidad para asegurar que ofrezcan los beneficios esperados a la sociedad. Preguntados cuales son los motivos que limitan una mayor frecuentación a los EVUPs, indican falta de tiempo (63\%), suciedad $(44,5 \%)$ y falta de seguridad y vigilancia $(45,7 \%)$ unido a encontrarse lejos de la vivienda $(29,1 \%)$ y presencia de mascotas sueltas (25,6\%). Además, consideran que el estado de conservación y grado de seguridad de los EVUPs es regular, e incluso malo o muy malo, como se aprecia en la Figura 3. La correlación del test de Tau-Kendall arroja que estas variables están significativamente relacionadas con la frecuentación: equipamiento $(r 2:-0,44 ; p=$ $0,00)$, cuidado $(r 2:-0,43 ; p=0,00)$, iluminación ( $r 2$ : $-0,47 ; p=0,00)$ y de seguridad $(r 2:-0,48 ; p=0,00)$.

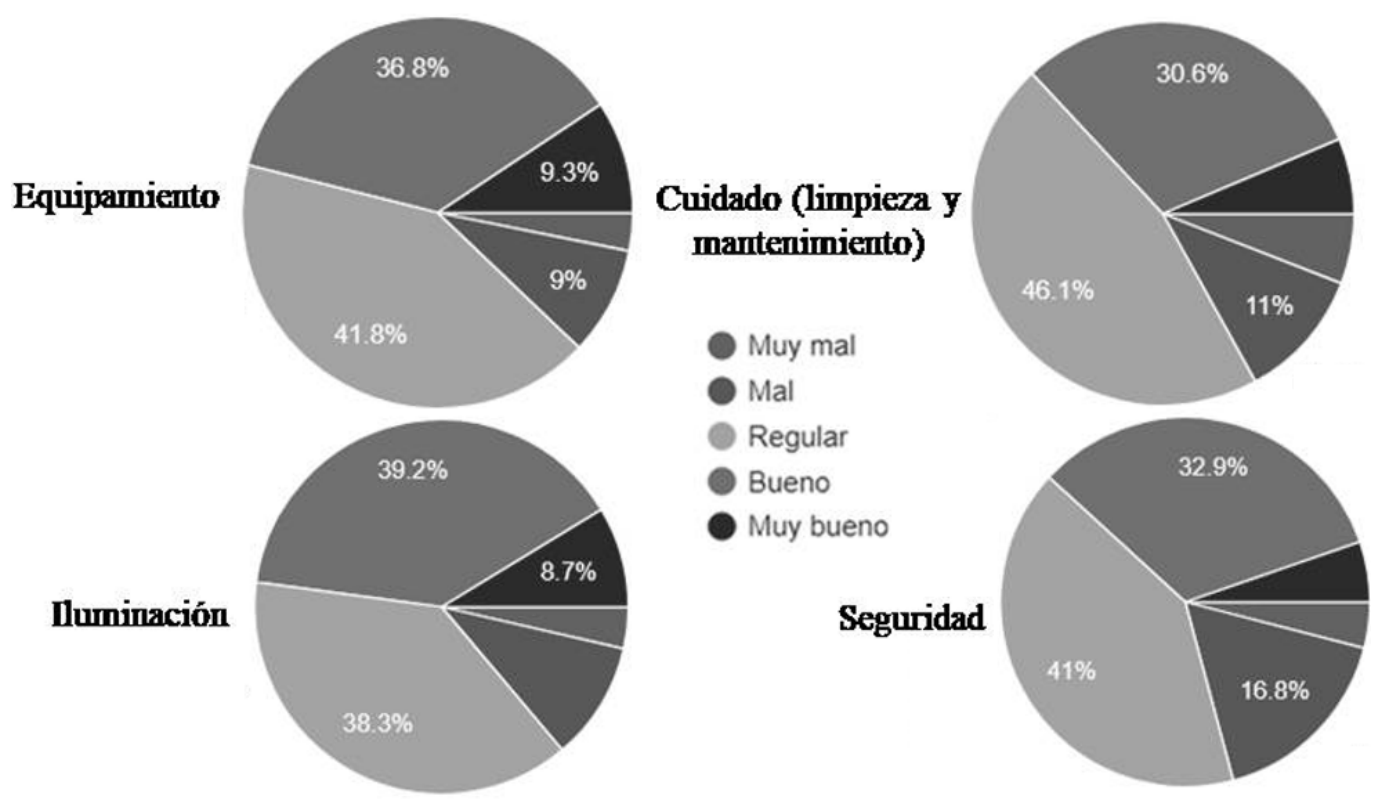

Figura 3. Respuestas sobre el estado y seguridad de los EVUPs más visitados según los Ciudadanos (N=1128) Fuente: elaboración propia.

Santana et al. (2010) hallaron una relación negativa entre EVUPs con señales de vandalismo o percibidos como inseguros y la realización en ellos de actividades como paseos o actividades deportivas. Por lo tanto, una mayor satisfacción de las condiciones del mobiliario, de la iluminación, del mantenimiento y una menor sensación de inseguridad incidirá en una mayor frecuentación del espacio público (Jasso, 2015).

El test de Tau-Kendall arroja que la frecuentación está significativamente relacionada con las variables distancia $(r 2: 0,46 ; p=0,00)$ y tipo de espacio verde $(r 2: 0,43 ; p=0,00)$, así como también con quien se va $(r 2: 0,43 ; p=0,00)$, el medio de transporte $(r 2: 0,44 ; p=0,00)$ y las actividades de ocio ( $r 2: 0,42 ; p=0,00)$. Una vez conocida estas relaciones, se analiza la importancia de las variables mediante la regresión MNL. La Tabla 9 muestra los resultados del test de bondad de ajuste y el valor del chi cuadrado indican validez del modelo, ya que resultan significativos. El coeficiente de Nagelkerke es aceptable al estar en torno a 0,5 y similar a los obtenidos en otros estudios (Jasso, 2015; Burrows et al., 2018). La mayoría de las variables son significativas, excepto la edad, género, tipo de vivienda, con quién, iluminación y cuidado del espacio verde. Se calculó que la tasa de precisión de la clasificación era del 49,9\% en comparación con el $34 \%$ solo por casualidad, lo que indica la utilidad del modelo (Pando y San Martín, 2004). 
de la Fuente - Espacios Verdes Urbanos Públicos: Ciudadanos y Técnicos Municipales Bases para una Gestión Sostenible

Tabla 9. Ajuste del modelo de regresión MNL.

\begin{tabular}{|c|c|c|c|c|}
\hline \multirow{2}{*}{ Modelo } & \multirow{2}{*}{$\begin{array}{l}\text { Criterios de ajuste de modelo } \\
\text { Logaritmo de la verosimilitud -2 }\end{array}$} & \multicolumn{3}{|c|}{ Pruebas de la razón de verosimilitud } \\
\hline & & Chi-cuadrado & gl & Sig. \\
\hline Sólo intersección & 2773,892 & & & \\
\hline Final & 2374,922 & 398,970 & 120 & 0,00 \\
\hline Nagelkerke r2 & 0,49 & & & \\
\hline Efecto & $\begin{array}{l}\text { Criterios de ajuste de modelo Logaritmo de la } \\
\text { verosimilitud }-2 \text { de modelo reducido }\end{array}$ & & & \\
\hline Intersección & $2374,922 a$ & 0,000 & 0 & \\
\hline Edad & 2394,096 & 19,174 & 12 & 0,08 \\
\hline Género & 2379,146 & 4,224 & 3 & 0,23 \\
\hline Tipo de Vivienda & 2391,302 & 16,380 & 9 & 0,05 \\
\hline Distancia & 2425,040 & 50,119 & 9 & 0,00 \\
\hline Tiempo & 2414,401 & 39,479 & 9 & 0,00 \\
\hline Con quien & 2382,482 & 7,560 & 6 & 0,27 \\
\hline Medio de transporte & 2399,142 & 24,220 & 12 & 0,01 \\
\hline Actividad de ocio & 2406,090 & 31,168 & 12 & 0,00 \\
\hline Equipamiento & 2402,757 & 27,836 & 12 & 0,00 \\
\hline Iluminación & 2391,667 & 16,746 & 12 & 0,15 \\
\hline Cuidado & 2386,498 & 11,577 & 12 & 0,48 \\
\hline Seguridad & 2426,009 & 51,087 & 12 & 0,00 \\
\hline
\end{tabular}

Fuente: Elaboración propia

Los resultados de la regresión MNL se presentan en la Tabla 10 con los tres modelos de frecuentación para las categorías: todas las semanas, varias veces al mes, algunas veces al mes; siendo la categoría de referencia: alguna vez o casi nunca. Las variables de género, con quien se va, medio de transporte y estado de conservación del equipamiento e iluminación no mostraron significancia en ninguno de los tres modelos.

Las personas que viven a menos de 500 metros de un EVUP tienen más de tres veces probabilidades de visitarlo semanalmente $(\operatorname{Exp} B=3,5)$ que quienes viven a más 3000 metros (categoría de referencia del análisis). Al examinar la frecuencia de aquellos que viven entre 500 metros y 1000 metros, el valor de ExpB fue de 3,5 para visitas semanales y de 3,2 para varias veces al mes. Mientras, aquellos que viven entre 1000 metros y 3000 metros, el valor de ExpB fue de 2,7 para varias veces por semana. Con estos resultados se puede establecer que una mayor probabilidad de visita sucede en los EVUPs más cercanos al domicilio donde viven los usuarios. Lo que se suma, que aquellos que frecuentan semanalmente los EVUPs tienen 3 veces más probabilidades de realizar actividades de ocio como caminar $(\operatorname{ExpB}=3,43)$ o reunirse con amigos (ExpB
$=3,35$ ) que ir a relajarse o descansar (categoría de referencia del análisis). Este patrón, también, fue significativo para el modelo de varias veces al mes; quienes tienen 2 veces más probabilidades de ir al espacio verde a caminar o reunirse con amigos ( $\operatorname{ExpB}=2,34$ y 2,24, respectivamente) que ir hacer otra actividad, como descansar.

Estas relaciones vienen a confirmar como la cercanía y actividades recreativas son variables relevantes a la hora de evaluar la frecuencia y uso de los EVUPs. Apkinar et al. (2016), así como Kruize et al. (2019) y Moran et al. (2020), demuestran la importancia de la proximidad de la vivienda a los EVUPs. De acuerdo con Apkinar et al, (2016) encontraron que cuanta más cerca estaba un espacio verde público de la casa de una persona, más se visitaba y más actividades al aire libre se hacían. Entre las razones principales para visitar el parque eran caminar o correr. Caminar, socializar fueron los principales motivos de la frecuentación a los EVUPs, y estos resultados se ratifican con los resultados de Giles-Corti et al. (2005), Lo et al. (2010) y Joseph et al. (2016), quienes encontraron que respirar aire fresco, caminar y socializar eran las razones principales por las que la gente visita los parques y plazas urbanas. 
Tabla 10. Modelos de regresión MNL, frecuencia a EVUPs más visitados ( $\mathrm{N}=1128)$.

\begin{tabular}{|c|c|c|c|c|c|c|c|}
\hline \multicolumn{2}{|c|}{ Frecuencia de visita* } & \multicolumn{2}{|c|}{ Todas las semanas } & \multicolumn{2}{|c|}{ Varias veces al mes } & \multicolumn{2}{|c|}{ Algunas veces al mes } \\
\hline Variables & Categorías & Sig. & ExpB & Sig. & ExpB & Sig. & ExpB \\
\hline \multirow[t]{5}{*}{ Edad } & $<18$ años & 0,04 & 0,270 & 0,57 & 0,380 & 0,17 & 084 \\
\hline & $18-30$ & 0,07 & 0,145 & 0,24 & 0,257 & 0,09 & 0,159 \\
\hline & $31-45$ & 0,03 & 0,104 & 0,21 & 0,241 & 0,10 & 0,164 \\
\hline & $46-65$ & 0,11 & 0,182 & 0,33 & 0,334 & 0,22 & 0,263 \\
\hline & +66 años & & & & & & \\
\hline \multirow[t]{4}{*}{ Tipo vivienda } & Casa/jardín & 0,25 & 0,636 & 0,54 & 0,775 & 0,58 & 0,798 \\
\hline & Casa/sin jardín & 0,03 & 0,365 & 0,22 & 0,543 & 0,74 & 0,853 \\
\hline & Apartamento con jardín & 0,61 & 1,342 & 0,55 & 1,435 & 0,78 & 1,183 \\
\hline & Apartamento sin jardín & & & & & & \\
\hline \multirow[t]{4}{*}{ Distancia } & $<500 m$ & 0,00 & 3,546 & 0,17 & 2,031 & 0,49 & 0,716 \\
\hline & $500-1000 \mathrm{~m}$ & 0,01 & 3,240 & 0,05 & 3,038 & 0,32 & 1,604 \\
\hline & $1000-3000 \mathrm{~m}$ & 0,10 & 2,255 & 0,03 & 2,755 & 0,18 & 1,902 \\
\hline & Más de 3000 m & & & & & & \\
\hline \multirow[t]{4}{*}{ Tiempo } & $<30$ & 0,16 & 0,386 & 0,04 & 0,250 & 0,10 & 0,331 \\
\hline & $1 \mathrm{~h}$ & 0,40 & 1,751 & 0,87 & 0,897 & 0,69 & 0,770 \\
\hline & 1- $2 \mathrm{hrs}$ & 0,11 & 2,841 & 0,47 & 1,638 & 0,40 & 1,730 \\
\hline & +2 horas & & & & & & \\
\hline \multirow[t]{5}{*}{ Actividad } & Caminar, pasear & 0,00 & 3,437 & 0,02 & 2,342 & 0,84 & 1,136 \\
\hline & Deporte & 0,72 & 1,338 & 0,86 & 1,162 & 0,19 & 0,316 \\
\hline & Jugar con hijos & 0,09 & 0,226 & 0,20 & 0,042 & 0,23 & 0,344 \\
\hline & Reunirse amigos & 0,03 & 3,351 & 0,01 & 2,242 & 0,99 & 1,129 \\
\hline & Relajarse, descansar & & & & & & \\
\hline \multirow[t]{5}{*}{ Cuidado } & Muy mal & 0,61 & 0,467 & 0,47 & 0,574 & 0,95 & 0,948 \\
\hline & Mal & 0,00 & 0,242 & 0,04 & 0,312 & 0,33 & 2,298 \\
\hline & Regular & 0,86 & 0,891 & 0,10 & 1,663 & 0,16 & 2,874 \\
\hline & Bueno & 0,45 & 1,669 & 0,14 & 1,866 & 0,55 & 1,528 \\
\hline & Muy bueno & & & & & & \\
\hline \multirow[t]{5}{*}{ Seguridad } & Muy mal & 0,02 & 0,269 & 0,02 & 0,189 & 0,41 & 0,245 \\
\hline & Mal & 0,01 & 0,367 & 0,04 & 0,225 & 0,15 & 0,183 \\
\hline & Regular & 0,22 & 5,702 & 0,80 & 1,467 & 0,16 & 0,196 \\
\hline & Bueno & 0,20 & 6,329 & 0,29 & 4,527 & 0,24 & 0,256 \\
\hline & Muy bueno & & & & & & \\
\hline
\end{tabular}

*: La categoría de referencia es: Alguna vez al mes o casi nunca.

Valor en negrita: $p$ (significación estadística) <0,05; Solo se muestran las variables que han resultados significativas en alguna categoría. $\operatorname{ExpB}=$ razón de probabilidades. Fuente: Elaboración propia

En el caso de la edad, los jóvenes menores de 18 años y adultos (31-45 años) fueron significativos para el caso de las visitas semanales, los coeficientes de probabilidades (ExpB) de 0.27 y 0.10 sugieren que ellos tienen un $73 \%$ y $90 \%$ menos de probabilidades de visitar los EVUPs que el grupo de edad $>65$ años (categoría de referencia del análisis). Este patrón no se repitió en los otros dos modelos de frecuentación. Probablemente, el hecho que los mayores frecuenten varias veces por semana los EVUPs pudiera ser, por ejemplo, para ir a caminar. El trabajo de Van Cauwenberg et al. (2017) muestra que cuanto más cerca viven personas mayores de un parque, mayor es la probabilidad de que lo visiten para pasear.
De acuerdo al tiempo de estancia, los resultados muestran que la significación sólo fue evidente en el segundo modelo. El coeficiente ExpB de 0,25 indica que quienes frecuentan un EVUP varias veces al mes tiene un $75 \%$ menos de probabilidades de pasar 30 minutos en él que aquellos que permanecen más de dos horas (categoría de referencia del análisis). Es decir, quienes visitan los EVUPs varias veces al mes se quedan por períodos más largos de tiempo. El trabajo de Bertam et al. (2017) muestra que los individuos pasan más tiempo en el parque si lo visitan los fines de semana que los días de semana. Madrid (2010) refuerza este hecho indicando que las personas durante los fines de semana disponen de más tiempo de ocio y realizan mayores desplazamientos para gastarlo en 
los EVUPs. En nuestro caso, no podemos asegurar que esto suceda durante los fines de semana. La categoría de fines de semana no fue considerada en este estudio quedando abierta su consideración para posteriores trabajos y así confirmar estas diferencias.

Según el tipo de vivienda, las personas que viven en casas sin jardín, los resultados fueron significativos para el caso de la frecuencia semanal, el coeficiente de probabilidad (ExpB) fue de 0.37. Los que viven en casas sin jardín tienen un $63 \%$ menos de probabilidades de visitar un espacio verde que el grupo de personas que viven en apartamentos sin jardín (categoría de referencia del análisis). Una mayor probabilidad de frecuencia hacia los EVUPs ocurra en personas que viven en apartamentos sin ningún tipo acceso a jardines privados. Esto estaría en la línea de Arnberger et al. (2017), quienes encontraron que los EVUPs que proporcionan un ambiente fresco con resguardo del sol aumentan la intención de visitarlo por personas que viven en departamentos, cuyas viviendas cerradas y pequeñas, les resulta agobiante en días calurosos.

Según el estado de conservación de los EVUPs, fueron significativos para el caso de las frecuencias semanales y varias veces al mes. Los coeficientes (ExpB) de 0.24 y 0,30 indican que los EVUPs mal cuidados tienen un $76 \%$ y $70 \%$ menos probabilidad de ser frecuentados que los EVUPs percibidos como muy bien cuidados (categoría de referencia de análisis). El mismo patrón siguió la seguridad percibida. Los coeficientes (ExpB) de 0,26 y 0,36 indican que los EVUPs percibidos como inseguros o muy inseguros tienen un $74 \%$ y $64 \%$ menos probabilidad de ser frecuentados que los EVUPs percibidos como muy seguros respectivamente (categoría de referencia de análisis). Esto viene a ratificar los trabajos ya comentados de Santana et al. (2010) y Jasso (2015).

En resumen, los resultados nos muestran que los Ciudadanos quieren tener EVUPs cercanos a sus viviendas en donde puedan caminar. Eso sí que estén bien cuidados y seguros. Dotar a los EVUPs de calidad es mantenerlos limpios, con equipamientos cuidados e iluminación en las zonas críticas; lo que favorece aumentar los niveles de satisfacción en las personas, por ende, una mayor frecuentación (Apkinar, 2016; ReyesPäcke et al., 2014). Una mayor frecuentación a los EVUPs, aumentaría la sensación de seguridad en las personas (Jasso, 2015). Tal como y como apunta
Jacobs (1961) una mayor presencia de personas en los espacios públicos ayuda a controlar el crimen y aumentar la seguridad ciudadana.

\section{Conclusiones}

Los resultados deben tomarse como una primera aproximación basada en la experiencia y actitud de las personas consultadas. Los análisis estadísticos de fiabilidad han sido positivos garantizando la validez de los resultados.

Se aprecia el consenso de los Técnicos Municipales consultados sobre la necesidad del papel de los EVUPs en la ciudad, concordando que una mayor cantidad y calidad de los mismos ayudaría a mejorar la calidad de vida urbana. De igual manera, se constata la carencia generalizada de normativas municipales que impliquen instrumentos de gestión y protección de los EVUPs. Al igual que en otros estudios, comprobamos que en los EVUPs existen necesidades y expectativas distintas entre Técnicos y Ciudadanos.

La mayor parte de los entrevistados usan los EVUPs cercanos al domicilio, accediendo preferentemente caminando, con visitas frecuentes, de preferencia por la tarde, con estancias superiores a una hora, yendo solo o acompañado, realizando actividades de ocio pasivas. La cercanía, frecuencia y el tiempo de permanencia son factores que nos muestran la importancia de los EVUPs para los usuarios entrevistados.

La accesibilidad y cercanía no son suficientes para que un espacio verde sea visitado. Es necesario que el EVUP posea una calidad suficiente para desencadenar en el ciudadano vínculos emocionales que le hagan sentirse satisfecho con su entorno. Esto muestra que los Ciudadanos quieren tener espacios cerca de ellos útiles y utilizables.

Por último, el trabajo pone de manifiesto la importancia que para mantener correctamente un EVUP es preciso conocer el uso y opinión de los Ciudadanos que los utilizan y de las personas que los gestionan. Se deben abrir los EVUPs a la ciudadanía a través de una participación activa, colaborativa y de co-creación; que los involucre en el destino de los EVUPs de sus barrios. De igual forma, los resultados también sugieren que mejorar la dotación de EVUPs debe venir acompañada de 
estrategias que eviten que estos lugares espacios se transformen en un foco de malestar para los vecinos.

En definitiva, surge la necesidad de tomar acciones para que los EVUPs sean mirados desde una perspectiva de planificación más amplia recogiendo el mosaico de realidades que componen la ciudad bajo un enfoque de responsabilidad e inteligencia territorial en un contexto de resiliencia y adaptación al cambio climático. Con objeto de buscar reforzar la funcionalidad y los beneficios que proporcionan los EVUPs hacia un modelo de ciudad más eficiente y sostenible. Por tal motivo, sugerimos que las relaciones entre la ciudadanía, los Técnicos Municipales y los EVUPs continúen estudiándose.

\section{Agradecimientos}

Se agradece al Dr. Cesar Pena Salmón de la Universidad Autónoma de Baja California (México) y a la Dra. Gloria Aponte de la Universidad de Ibagué, (Colombia) por ayudar en la corrección del cuestionario.

\section{REFERENCIAS}

Akbari, H. (2002). Shade trees reduce building energy use and $\mathrm{CO} 2$ emissions from power plants. Environmental Pollution, 116 (1). doi: https://doi.org/10.1016/S0269-7491(01)00264-0.

Apkinar, A. (2016). How is quality of urban green spaces associated with physical activity and health?. Urban Forestry \& Urban Greening, 16, 76-83. doi: https://doi.org/10.1016/j.ufug.2016.01.011

Arnberger, A., Brigitte, E., Eder, R., Ebenberger, M., Wanka, A., Kolland, F., Wallner, $P$.

y Hutter, H. (2017). Elderlyresident's uses of and preferences for urban green spaces during heat periods. Urban Forestry \& Urban Greening. 21. doi: https://doi.org/10.1016/j.ufug.2016.11.012

Barrón, D. (2015). Áreas verdes públicas lineales y su aporte a la integración social en Santiago de Chile (Tesis de maestría). Universidad de Chile.

Bertram, C., Meyerhoff, J., Rehdanz, K. y Wustermann, H. (2017). Differences in the recreational value of urban parks between weekdays and weekends: A discrete choice analysis. Landscape and Urban Planning, 159, 5-14. doi: https://doi.org/10.1016/j.landurbplan.2016.10.006

Brenner, L. y De la Vega, A. (2014). La gobernanza participativa de áreas naturales protegidas. El caso de la Reserva de la Biosfera El Vizcaíno. Región y Sociedad, 26(59): 183- 213. Recuperado de: http://www. scielo.org.mx/scielo.php?script=sci arttext\&pid=S1870-39252014000200006

Burel, F. y J. Baudry (2002). Ecología del paisaje. Barcelona, España: Mundi Prensa

Burrows, E., O'Mahony, M. y Geraghty, D. (2018). How Urban Parks Offer Opportunities for Physical Activity in Dublin, Ireland. International. Journal of Environmental Research and Public Health. 15(4). doi: 10.3390/ ijerph15040815

Centelles i Portella, J. (2006). El buen gobierno de la ciudad. Estrategias urbanas y política regional. La Paz, Bolivia: Plural editores.

Coles, R. y Bussey, S. (2000). Urban forest landscapes in the UK: progressing the social agenda. Landscape and Urban Planning, 52, 181-188. doi: https://doi.org/10.1016/S0169-2046(00)00132-8

Cornejo, C. (2016). Elementos para la participación ciudadana en la fiscalización pública: una aproximación desde la experiencia de la contraloría general de Paraguay (2006-2014) (Tesis de maestría). Universidad de Georgetown. 
de la Fuente - Espacios Verdes Urbanos Públicos: Ciudadanos y Técnicos Municipales Bases para una Gestión Sostenible

Corral-Verdugo, V. y Pinheiro, J. (2004). Aproximaciones al estudio de la conducta sustentable. Medio Ambiente y Comportamiento Humano, 5(1-2), 1-26.

De la Fuente-de Val, G. (2002). Análisis de escenarios paisajísticos y medidas de calidad escénica. Estudio de casos: la Sierra de Guadarrama (Madrid, España) y la precordillera Andina de Santiago (Chile) (Tesis doctoral). Universidad Autónoma de Madrid, Madrid.

De la Fuente-de Val, G. (2020). Informe estudio sobre el paisaje urbano en ciudades de América Latina y el Caribe. Recuperado de: https://cutt.ly/wkPk4mB

Emakunde. (2014). La evaluación de impacto en función del género en el medio en el medio ambiente. Departamento de Medio Ambiente y Política Territorial del Gobierno Vasco.

Faggi, A. y Perepelizin, P. (2006). Riqueza de aves a los largo de un gradiente de urbanización en la ciudad de Buenos Aires. Revista Museo Argentino, Ciencias Naturales, 8 (2), 289-297

Falcón, A. (2007). Espacios verdes para una ciudad sostenible Planificación, proyecto, mantenimiento y gestión. Barcelona, España: Gustavo Gili.

FAO. (2014). Urban and Peri-urban Agriculture in Latin America and the Caribbean: Compendium of case studies.

Figueroa, I. (2009). Conectividad y accesibilidad de los espacios abiertos urbanos en Santiago de Chile (Tesis de maestría). Pontificia Universidad Católica de Chile, Santiago.

Flores, R. (2012a). Incorporando desarrollo sustentable y gobernanza a la gestión y planificación de áreas verdes urbanas. Frontera norte, 24(48). Recuperado de: http://www.scielo.org.mx/scielo. php?script=sci arttext\&pid=S0187-73722012000200007\&lng=es\&tlng=es.

Flores, R. (2012b). Análisis de los gustos recreativos en el Parque Ambiental Bicentenario de Metepec, Estado de México (México). PASOS. Revista de Turismo y Patrimonio Cultural, 10(3), 312-329. doi: https://doi. org/10.25145/j.pasos.2012.10.038

Forsyth, A. (2003). People and urban green areas: perception and use. Design centre for American Urban Landscape. University of Minnesota.

García, C., Paiva, P., Bruck, J. y Sousa, R. (2021). Distribution of urban green spaces: Comparative analysis between cities in different countries. Ornamental Horticulture, 27(1), 8-19. doi: https://dx.doi. org/10.1590/2447-536x.v27i1.2151

Giles-Corti, B., Broomhall, M., Collins, C., Douglas, K., Lange A. y Donovan, R. (2005). Increasing walking. How important is distance to, attractiveness and size of public open space?. American Journal of Preventive Medicine, 28, 169-176. doi: https://doi.org/10.1016/j.amepre.2004.10.018

Gogiel, G. (2011). Conciencia social y ahorro de agua doméstica según las diferentes tipologías urbanas. (Tesis de maestría). Universitat Politècnica de Catalunya, España.

Gómez, J. y Mesa, A. (2015). Análisis de los modos de acceso y los patrones de uso de la población respecto a los espacios verdes urbanos, como base para su planificación. Urbano, 18(32), 38-49. Recuperado de: http://revistas.ubiobio.cl/index.php/RU/article/view/1995

Gómez-Gonçalves, A. (2013). El valor funcional de la estructura verde urbana aportación desde el estudio de los espacios verdes de la ciudad de Braganza (Portugal) (Tesis Doctoral). Universidad Politécnica de Madrid, Madrid. 
Gómez-Gonçalves, A., Sánchez Hernández, J. y Ceballos, A. (2018). El impacto de las políticas de austeridad en los espacios verdes urbanos según la percepción de los usuarios. Estudio de caso en tres ciudades españolas. Boletín de la Asociación de Geógrafos Españoles, 77, 398-427. doi: http://dx.doi.org/10.21138/ $\underline{\text { bage. } 2546}$

González, E. (1995). Manual sobre participación y organización para la gestión local. Medellín, Colombia: Ediciones Foro Nacional.

Handley, J., Pauleit, S., Slinn, P., Barber, A., Baker, M. y Jones, C. (2003). Accessible Natural Green Space Standards in Town and Cities: A Review and Toolkit for their Implementation. Peterborough UK: English Nature Report Number 526.

Harnik, P. (2010). Urban Green: Innovative Parks for Resurgent Cities. Washington, USA: Island Press.

Harnik, P. y Crompton, J. (2014). Measuring the total economic value of a park system to a community. Managing Leisure, 19(3), 1-24. doi: https://doi.org/10.1080/13606719.2014.885713

Honey-Roses, J., Anguelovski, I., Bohigas, J., Chireh, V. K., Mr., Daher, C., Konijnendijk, C. y Nieuwenhuijsen, M. (2020). The impact of COVID-19 on public space: an early review of the emerging questions: design, perceptions and inequities. Cities \& Health. doi: 10.1080/23748834.2020.1780074

IBM Corp. Released (2020). IBM SPSS Statistics for Windows, Version 27.0. Armonk, NY: IBM Corp Jacobs, J. (1961). The Dead and Life of Great American Cities. Nueva York, USA: Random House.

Jasso, C. (2015). Porque la gente se siente insegura en el espacio público. Política pública de prevención situacional del delito (Tesis doctoral). Centro de Investigaciones y Docencia Económicas, Ciudad de México.

Joseph, R. y Maddock, J. (2016). Observational park-based physical activity studies: A systematic review of the literature. Preventive Medicine, 89, 257-277. doi: 10.1016/i.ypmed.2016.06.016

Karade, R., Kuchi, V. y Salma, Z. (2017). The Role of Green Space for Sustainable Landscape Development in Urban Areas. International Archive of Applied Sciences and Technology, 8 (2), 76-79. doi: 10.17660/ ActaHortic.2017.1181.9

Kawgan-Kagan, I. (2020). Are women greener than men? A preference analysis of women and men from major German cities over sustainable urban mobility. Transportation Research Interdisciplinary Perspectives, 8. doi: https://doi.org/10.1016/j.trip.2020.100236

Kinne, O. (1997). Ethics and ecoethics, en Marine Ecology Progress, Germany: International Ecology Institute.

Krellenberg, K., Jordán, R., Rehner, J. Schwarz, A. Infante, B., Barth, K. y Pérez A. (2013). Adaptación al cambio climático en megaciudades de América Latina Red Regional de Aprendizaje. Informe proyecto: Climate Adaptation Santiago (CAS).

Kruize, H., Van der Vliet, N., Staatsen, B., Bell, R., Chiabai, A., Muiños, G., ... Stegeman, I. (2019). Urban Green Space: Creating a Triple Win for Environmental Sustainability, Health, and Health Equity through Behaviour Change. International Journal of Environmental Research and Public Health, 16: 4403. doi: https://doi.org/10.3390/ijerph16224403

Laurie, M. (1983). Introducción a la arquitectura del paisaje. Barcelona, España: Editorial Gustavo Gili. 
de la Fuente - Espacios Verdes Urbanos Públicos: Ciudadanos y Técnicos Municipales Bases para una Gestión Sostenible

Lo, A. y Jim, C. (2010). Willingness of residents to pay and motives for conservation of urban green spaces in the compact city of Hong Kong. Urban Forestry \& Urban Greening, 9, 113-120. doi: https://doi. org/10.1016/j.ufug.2010.01.001

Low, S., Taplin, D. y Scheld, S. (2005). Rethinking Urban Parks. Public Space and Cultural Diversity. Texas, USA: The University of Texas Press.

Madrid, A. (2010). Perfil, actividades y grado de satisfacción de los usuarios en parques urbanos. Estudio en tres parques urbanos, Santiago, Chile. (Tesis de maestría). Magíster en Gestión y Planificación Ambiental. Santiago: Facultad de Ciencias Forestales y Conservación de la Naturaleza, Universidad de Chile.

Mäkinen, K. y Tyrväinen, L. (2019). Teenage experiences of public green spaces in suburban Helsinki, Urban Forestry\& Urban Greening, 7(4), 277-289. doi: https://doi.org/10.1016/j.ufug.2008.07.003

Márquez, F. (2009). Diseño participativo de espacios verdes públicos. Cuadernos del Centro de Estudios de Diseño y Comunicación, 30, 155-166. doi: https://doi.org/10.18682/cdc.vi30.1514

Marselle, M., Bowler, D., Watzema, J., Eichenberg, D., Kirsten, T. y Bonn, A. (2020). Urban street tree biodiversity and antidepressant prescriptions. Science Reports, 10, 22445. doi: https://doi.org/10.1038/s41598-020$\underline{79924-5}$

Mejía, G. y Gómez, R. (2016). El impacto social en el parque público General Esteban Baca Calderón "La Loma" de Tepic, Nayarit, México. Revista Iberoamericana de las Ciencias Sociales y Humanísticas, 5 (9). https://dialnet.unirioja.es/servlet/articulo?codigo $=5433065$

Mena, C., Fuentes, E., Ormazábal, Y., Palomo-Vélez, G. y Palomo, I. (2015). Role of access to parks and markets with anthropometric measurements, biological markers, and a healthy lifestyle. Journal of Environmental Health Research, 25, 373-383. doi: 10.1080/09603123.2014.958134

Mengbing, D. y Xiaoling, Z. (2020). Urban greening: A new paradox of economic or social sustainability?, Land Use Policy, 92. doi: https://doi.org/10.1016/j.landusepol.2020.104487

Moran, M., Rodríguez, D., Cotinez-O'Ryan, A. y Miranda, J. (2020). Park use, perceived park proximity, and neighborhood characteristics: Evidence from 11 cities in Latin America, Cities, 105. doi: https://doi. org/10.1016/j.cities.2020.102817

Nilsson, K., Randrup, T. y Tvedt, T. (1997). Aspectos tecnológicos del Enverdecimiento Urbano. En Krishnamurthy L. y J. Rente Nascimento, (Eds.), Áreas Verdes Urbanas en América Latina y El Caribe (pp. 39-81). México, Universidad Autónoma Chapingo,

Nunnally, J. (1978). Psychonmetric Theory. Nueva York, USA: McGraw-Hill Book.

Palomo, P. (2003). La Planificación Verde en las Ciudades. Barcelona, España: Gustavo Gili.

Pando, V. y San Martin, R. (2004). La regresión logística multinominal. Cuaderno de la Sociedad Española de Ciencias Forestales, 18, 323-327. doi: https://doi.org/10.31167/csef.v0i18.9478

Pons, B. (2016). La infraestructura verde. Base de la Resiliencia Urbana. Estrategias para la regeneración de corredores fluviales urbanos de Banco Interamericano de Desarrollo (Tesis doctoral). Universidad Politécnica de Madrid, Madrid. 
Rey, G.; Barrigón, J.; Montes, D. y Atanasio, P. (2018). Relationships among satisfaction, noise perception, and use of urban green spaces, Science of the Total Environment, 624: 438-450. doi: https://doi.org/10.1016/j. scitotenv.2017.12.148

Reyes-Päcke, S., De La Barrera, F., Dobbs, C., Spotorno, A. y Pavés, C. (2014). Costos de mantención de las áreas verdes urbanas en Chile. Informe final. Centro de Políticas Públicas, Departamento de Ecosistemas y Medio Ambiente, Universidad Católica de Chile.

Roy, S., Byrne, J. y Pickering, C. (2012). A systematic quantitative review of urban tree benefits, costs, and assessment methods across cities in different climatic zones. Urban Forestry \& Urban Greening, 11 (40). doi: https://doi.org/10.1016/j.ufug.2012.06.006

Sahraoui, Y., De Godoy, C., Benot, M., Revers, F., Salles, D., van Halder, I., ... Carassou, L. (2021). Integrating ecological networks modelling in a participatory approach for assessing impacts of planning scenarios on landscape connectivity, Landscape and Urban Planning, 209. doi: https://doi.org/10.1016/j. landurbplan.2021.104039

Salvo, D., Sarmiento, O., Reis, R., Hino, A., Bolivar, M., Lemoine, P. y PrattWhere, M. (2017). Where Latin Americans are physically active, and why does it matter? Findings from the IPEN-adult study in Bogota, Colombia; Cuernavaca, México; and Curitiba, Brazil, Preventive Medicine, 103, S27-S33. doi: 10.1016/j. ypmed.2016.09.007

Sanahuja, J. (2013). Valoración medioambiental de los árboles en los espacios verdes urbanos aplicación sobre el municipio de Sant Cugat del Vallès. (Tesis de Maestría). Universidad politécnica de Cataluña.

Santana, P., Costa, C., Santos, R., y Loureiro, A. (2010). O papel dos Espaços Verdes Urbanos no bemestar e saúde das populações. Revista de Estudios Demográficos, 48, 6-33. Recuperado de: https:// www.researchgate.net/publication/270450966 O papel dos Espacos Verdes Urbanos no bemestar e saude das populacoes

Scopelliti, M., Carrus, G., Adinolfi, C., Suarez, G., Colangelo, G., Lafortezza, R. y Sanesi, G. (2016). Staying in touch with nature and well-being in different income groups: The experience of urban parks in Bogotá, Landscape and Urban Planning, 148, 139-148. doi: https://doi.org/10.1016/j.landurbplan.2015.11.002

Stephens, R. (2014). Espacio Urbano, Delito y Prevención. Ontario, Canada: División de Planificación Urbana.

Stessens, P., Canters, F., Huysmans, M., Khan, A. (2020). Urban green space qualities: An integrated approach towards GIS-based assessment reflecting user perception, Land Use Policy, 91. doi: https:// doi.org/10.1016/j.landusepol.2019.104319

Ugolini, F., Massetti, L. Calaza-Martínez, P., Cariñanos, P., Dobbs, C., Krajter C., ... Sanesi, G. (2020). Effects of the COVID-19 pandemic on the use and perceptions of urban green space: An international exploratory study. Urban Forestry \& Urban Greening, 56. doi: https://doi.org/10.1016/j.ufug.2020.126888

Van Cauwenberg J., Cerin E., Timperio A., Salmon J., Deforche B. y Veitch J. (2017). Is the association between park proximity and recreational physical activity among mid-older aged adults moderated by park quality and neighborhood conditions?. International Journal of Environmental Research and Public Health. 14:192. doi: https://dx.doi.org/10.3390\%2Fijerph14020192

Vargas, A. y Roldán, P. (2018). Ni muy cerca ni muy lejos: parques urbanos y bienestar subjetivo en la ciudad de Barranquilla, Colombia. Lecturas de Economía, 88, 183-205. doi: https://doi.org/10.17533/udea. le.n88a06 
de la Fuente - Espacios Verdes Urbanos Públicos: Ciudadanos y Técnicos Municipales Bases para una Gestión Sostenible

Walker, C. (2004). Understanding park User ship. The Urban Institute and the Wallace Foundation, Washington, D. C. United States of America.

Whyte, W. (1980). The Social Life of Small Urban Spaces. Nueva York: The Project for Public Spaces Inc.

Yang, L., Ho, J., Wong, F., Chang, K., Long, K. Sing, M., ... Siu, J. (2020). Neighbourhood green space, perceived stress and sleep quality in an urban population, Urban Forestry \& Urban Greening, 54. doi: https://doi. org/10.1016/j.ufug.2020.126763 